\title{
When can we treat Likert type data as interval? [Project Proposal]
}

\section{Miguel Alejandro A. Silan}

Author's note: This is a paper that was supposed to be an independent research project. Somehow or another, it fell to the wayside. Since it'll be some time before I get back to it, I'm releasing this working review/proposal paper to the public in case it helps anyone wanting to answer the same problems. For correspondence: MiguelSilan@gmail.com

This paper lays down key issues in ordinality, and a broad-sketch approach to data simulation to answering when (i.e. which data conditions) likert type data can be considered interval rather than ordinal

\section{Is there really a problem?}

The appropriateness of using 'standard' linear metric models (t-, F-test, and regression variants) on ordinal data, including Likert and Likert type items and scales, has been debated upon almost as early as Likert scales themselves has been introduced to Psychology (Likert, 1932; Stevens, 1946; Knapp, 1990). However, this almost century old debate has focused largely on the theoretical issue of what what constitutes an ordinal scale versus what constitutes an interval scale; and there is unproportionately less work that tackles the problem in an empirical/data simulation approach. In this section, we will trace the arguments from both camps on the defensibilty/detriment of using metric models on ordinal and Likert type data.

THERE IS NO PROBLEM BECAUSE... many believe that a simple workaround to the problem of ordinality in Likert items is simply not to analyze items, but to analyze Likert scales (Carifio \& Perla, 2008). That is, while one item is ordinal, the sum or the average of several Likert items represent now a continuous measure. A contrived but analog case: a single binary true/false question is obviously non-continuous, but a set containing the total scores in 100 true/false questions now look more interval than categorical. In fact, Likert (1932) himself set the heuristic that enough items should be added to attain sound scale reliability; and that Likert type data can be considered interval "particularly if the scale meets the standard psychometric rule-of-thumb criterion of comprising at least eight reasonably related items" (Carifio \& Perla, 2008)

However, even with this ideal case, (1.) the question becomes how many items are needed to get to the 'safe zone' of intervality; and does the number of categories/response options matter? For example, does a scale with items having 11 anchor points need fewer items to be considered interval than a scale with items having 5 anchor points? Is a single item visual analog scale, with a bounded, but infinite points, considered to be interval? And given how common 'short form scales' are in the field, are there systematic errors of using them as dependent variables that we can correct for? Further, (2.) despite their face value, there is no guarantee that this summed (or averaged) total score -which now represent a finer-grained scale- is truly equally spaced. For the IQ test for example, are we really sure that the distance between 80 and 90 is the same as between 120 and 130? [Consider for example a true interval measure of height, we can say that John is 10 inches shorter than Mark. Similarly for IQ, can we say that John is 10 units dumber than Mark?] And if the distances are not equal, will using IQ as a dependent variable in a regression upwardly or downwardly bias effect size measures? Will it systematically inflate or deflate false positive errors? (3.) In a well-considered and recent simulation work, Liddell and Kruschke (2018) show that even pooling multiple ordinal items together do not solve the estimation and significance problem of using standard linear models in sums of ordinal items. 
THERE IS NO PROBLEM BECAUSE... Likert type items and scales -despite being conceded as ordinalare defended to be appropriate for metric model analysis because they are usually thought to measure latent variable constructs, which (implicitly thought or explicitly modelled) the researchers believe to be continuous (Kampen and Swyngedow, 2000). While tempting to be considered as a solution to ordinality, this again run into its own issues. Applying standard linear regression models when the dependent variable is ordinal is not appropriate because 1.) using standard linear regression, instead of using latent variable methods (CFA, EFA, SEM) has the strong assumptions of having no measurement error (Kline, 2015) and that the Likert type items that constitute the scale are unidimensional in the first place and all are valid indicators of the latent variable. More importantly 2.) A respondent may have one set arguments for agreeing, and another for not-agreeing. Thus the observed variability in the individuals' scores may be due to several variables, which in themselves may or may not be continuous (Kampen \& Swyngedouw, 2000). And unfortunately, 3.) even in the ideal condition that the Likert scale DV represent only one underlying variable, which is continuous and normal; if the scale of the response options are wider than the true underlying scale of the variable, then analysis using metric models will affect both effect size estimation and significance testing (Liddell \& Kruschke, 2018) which is not the case for when these are analyzed taking into account their ordinal-categorical nature, such as when using ordered-probit, as well as other ordinal analysis including logistic regression (for a review: Agresti, 2010)

THERE IS NO PROBLEM BECAUSE... Lastly, the issue of Likert items being ordinal is often ignored because classic 'robustness' literature show that the t- and F-tests are robust against certain violations, including the limiting of the number of response options even with small samples (Heeren \& D'Agostino, 1987) and heterogenous variance (Hsu \& Feldt, 1969; if the sample sizes are equal). That is, researchers fall into pragmatic justification when using linear metric models on ordinal data - while not 'purely'/technically "appropriate", because the effects on the statistical inference wouldn't be severe. As Carifio and Perla (2007) one of the staunchest defenders of metric analyses on Likert scale says that the F "is not made of glass". Norman (2010) summarizes this, stating "Parametric statistics can be used with Likert data, with small sample sizes, with unequal variances, and with non-normal distributions, with no fear of 'coming to the wrong conclusion'"'

On the other half of the divide are methodologists who do argue

THERE IS A PROBLEM BECAUSE... (Marcus-Roberts\& Roberts, 1987) asserts that standard linear models on ordinal data represent meaningless statistics. It's not that hypothesis tests (of t-, F-, regression) are meaningless, its what it's tested on. That is, the means themselves of ordinal data are meaningless values (or more technically, the inference from the means in meaningless). One may always calculate a mean on a set of numbers, but because it did not have meaning in the first place, the hypothesis testing it are also rendered meaningless. However, while the arithmetic mean of the Olympic medals across the years $\left(1^{\text {st }}, 2^{\text {nd }}, 3^{\text {rd }}\right)$ seem to obviously not represent anything of value, the arithmetic mean of several Likert items seem like it is not completely devoid of meaning. Thus, there seems to be a different degrees (or even different kinds) of ordinality, all of which share the property of a set that only rank order is assured. Kampen and Swyngedouw (2000) lists down five different kinds of ordinalities, some of which represent an underlying continuous variable, some of which in themselves are just ordered categories. In other words, the arithmetic mean seem to be a relevant measure for some, but not for other, kinds of ordinality 
THERE IS A PROBLEM BECAUSE... In a well considered study, Liddell and Kruschke (2018) strongly present that in some instances (in some data conditions) the standard linear models distort both the effect size and the statistical significance when used in ordinal data. Conceptually, this should be when there is a huge mismatch between the scale of the (observable) response options and the scale of the underlying variable; as well as when the underlying variability of the latent mean differ between two groups. A detailed look at their simulations show that this mismatch leads to an observable floor and ceiling effects in the data; conditions which Agresti (2002) note to be when linear models are most vulnerable to be misleading. ${ }^{1}$

THUS knowing that there are data conditions that allow linear models to be robust against assumptions (including the assumption of continuous scale), and conditions where these largely affect statistical inference, the problem isn't so much as "is standard linear models appropriate to apply on ordinal data" but rather "when are standard linear models appropriate to apply on ordinal data?". Which, essentially is similar to asking "when can we treat Likert type data as interval?"

Further, applied data analysis as commonly done in psychological research is a chimaera of tools, epistemologies and assumptions many of which are implicitly held. This project aims to contribute in a meta-methodological level (especially in knowing how and when our methods work and fail) by conducting how different models work in different data conditions, which will allow us to inquire which particular model-data combinations are defensible, and making explicit which assumptions are tenable.

\section{HOW CAN WE SOLVE A PROBLEM LIKE ORDINALITY?}

From the discussion above, it's easy to get lost in the myriad of issues surrounding ordinality, which Likert type items (and arguably, Likert type scales) essentially represent. To facilitate thinking about the issue, its helpful to see the issue as both a MEASUREMENT and ANALYSIS problem. That is, the threat to inference from the statistical analysis begins early on in the process.

\section{MEASUREMENT}

Psychologists are usually interested in constructs and attributes that cannot be observed directly; including attitudes, traits, personality and other psychological variables (self-esteem, learning, mental disorders etc.). Because these attributes cannot be directly observed, by-products of these attributes are measured - whether as behaviors, as answers to pen-and-paper items. neural signals and son on. Thus, how measurement is conducted becomes foundational. Likert(-type) items and scales are one of the most common ways of measuring these attributes: often asking individuals to rate their agreement to particular items from strongly disagree to strongly agree commonly with 5 to 11 anchor points; and many scales are constructed that presents related items seeking to describe or isolate one variable.

The philosophical grounding that these pen-and-paper indicators $L$, actually do represent some attribute $A$, is the position of the representational measurement theorists (Kampen \& Swyngedouw, 2000), the most influential of which is Stevens (1946), who popularized the four (4) measurement levels: nominal, ordinal, interval, ratio. Here, measurement is taken to be the assignment of numbers according to some rule (Borsboom, 2018) however for the representationalists, there must be a one-

\footnotetext{
${ }^{1}$ Earlier studies by Hsu \& Feldt (1969) and Heeren \& D'Agostino (1987) who argue for the robustness of t- and Ftest examine the effects of using only 4-5 response options but they assume equal spacing between these response options; with all distributions being unimodal, with no severe skew or kurtosis
} 
to-one correspondence between $L$ and $A$; thus $L$ needs to be isomorphic, but not identical to $A$ each measurement scale. Put another way, if $L$ and $A$ are isomorphic, then by knowing the relationship between numbers ( $\mathrm{L} 1 \neq \mathrm{L} 2, \mathrm{~L} 1<\mathrm{L} 2, \mathrm{~L} 1 n$ units $<\mathrm{L} 2)$ we also find out the relationship between the attributes. For the representationalists, the difference between the scale levels is determined by the set of transformations that leave the isomorphism intact. For example, for ordinal scale this is any transformation which leaves the rank order intact, while for nominal this is pretty much any transformation aside from the equality of the values. That is, for the nominal level, "male" and

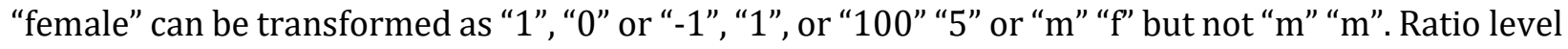
meanwhile, having a non-arbitrary zero and equal intervals have almost no transformation that can leave the isomorphism intact.

However, a fundamental criticism of the representational approach is that for many of the topics we are interested in, it is not empirically possible to know whether indicator $L$ is isomorphic of attribute $A$. Because the attribute is unobservable, the way to know about $A$ is to measure it in the first place! Because $A$ is unobservable, so then is the correspondence between $L$ and $A$, and so the needed link to know the scale of measurement is absent. Therefore, it is also not empirically possible to verify what the scale of measurement for is (Kampen \& Swyngedouw, 2000) What is often done is to classify according to rational and not empirical grounds: whether the scale is nominal (response options indicate differences), ordinal (response options indicate rank order), interval (equal distances between response options) and ratio (presence of a true zero point); and to give prototypic examples of each (jersey numbers, Olympic ranking, Celsius, kelvin respectively). As Nunnaly (1967) notes “... no one has made it clear on what types of evidence would justify the assumption of a particular type of scale"

The operational measurement theorists position tries to get away from the first criticism of the unobservable $A$ by noting that the existence of some underlying $A$ does not matter; indicators $L$ themselves are identical to the attributes $A$. That is, the scores on the scale represent nothing more beyond it ${ }^{2}$ (Nunnaly \& Bernstein , 1994). However it does not solve them more pressing problem of verifying the scale measurement (Kampen \& Swyngedouw, 2000). Stevens (1946) differentiates the levels, the transformations that can be done, and how they might be analyzed; however how to choose the measurement level of any particular/given scale is not presented. As Knapp (1990) says:

First, there are no agreed-upon rules for determining whether a particular scale is ordinal, less than ordinal, or more than ordinal. Consider, for example, the scale consisting of the following categories: never, seldom, frequently, always. Most researchers would agree that those four categories are mutually exclusive and ordered, in the sequence provided. Assigning the numerals 1, 2, 3, 4 to the four categories seems to be a reasonable thing to do; but any order preserving transformation... would seem to be equally admissible, and most would agree that such a scale is indeed ordinal. But suppose that the ordinal categories were: never, occasionally, sometimes, always. There would be little agreement among judges (and grammarians) about the relative placement of the two middle categories, thus producing a less-than-ordinal scale.

\footnotetext{
${ }^{2}$ Much of the representational position is forwarded in the classical measurement theory; however, Item response theory which include the use of probabilistic models including IRT and Rasch models to measure some subject parameter (theta), as well as other test parameters like difficulty and guessing \& assumes that these parameters determine the likelihood of the given responses in the items. (Nunnally \& Bernsetin, 1994) In this sense, IRT models handle ordinal data better as they take into account response options, and patterns of responses to extract latent variable parameters.
} 
Again, indicating that several different kinds of ordinality might exist, and these different kinds of ordinality might have different "admissible statistics".

\section{SCALING / SCORING PROCEDURE / NUMERICAL MAPPING}

When creating a Likert item or scale; with the five response options strongly disagree to strongly agree what should the numerical mapping be? Should there disagrees be negative? $(-2,-1,0,1,2)$ or should the numbers start at 0 ? Start at 1 ?

Strictly, if taken to be essentially ordinal, any monotonically increasing transformation can be done on the numerals corresponding to the response options, without 'breaking' the isomorphism between $L$ and $A$ (Stevens 1946, Borsboom 2018). That is, any transformation that lets the last option be greater than the second last option, which should be greater than the $3^{\text {rd }}$ last option etc. And this doesn't need to be equally spaced so strictly $(1,2,3,4,5)$ and $(1,5,10,20,100)$, and $(1,3,10,18,100)$ are valid transformations of the original numerical mapping of the ordinal response options (and thus, the listed ordinal data) because only the rank order is and should be guaranteed in the first place. The median in any case would be consistent across the monotonic transformations ${ }^{3}$, while the mean would vary wildly. These same means are crucial for standard linear models, but not for ordinal types of analyses.

In fact, it seems that the common numerical mapping scheme $(1,2,3,4,5)$ because the numbers have equal intervals might be the psychological crutch that makes researchers complacent about the distance between the underlying response options. And to reiterate, even at this even at this stage of measurement, choices abound and there are no foolproof guides one way or another.

\section{ANALYSIS}

Whatever measurement philosophy is upheld when designing the tests and collecting the data, data analysis itself can be done independent of the measurement theory and procedure that precedes it. As the common mantra, taken from Lord (1953) "'the numbers don't remember where they came from". Thus, Lord upholds that data can be subjected to parametric statistics and hypothesis tests, even though the scales of measurement that the data come from are anywhere from nominal, ordinal, interval or ratio. Whether these produce meaningful inference is another matter, but as long as they meet the distributional assumptions then the measurement level is a non-concern.

For standard linear models (t-, F- and regression ) these assumptions include: 1.) linearity 2.) that errors are independent and identically distributed, 3.) there is homogeneity of variance 4.) error is normally distributed 5.) the sampling distribution of the parameter is normally distributed (Keppel and Wickens, 2004; Field \& Wilcox, 2017; Field 2017) It is also commonly held that the data for the dependent variable should be continuous, as seen in the options of common statistical programs (SPSS, JASP, JAMOVI) although this is not technically correct (Knapp, 1990) and again the contentious assumption that only interval level and ratio level measured data are appropriate.

This is where the 'robustness' literature comes in, as discussed on the first section (Hsu \& Feldt 1969; Heeren \& D'Agostino, 1987 Liddell \& Kruschke, 2018 as well as Field and Wilcox, 2017; Wilcox, 2011 among many others). That is, how 'robust' or resistant to violations of assumptions different

\footnotetext{
${ }^{3}$ Techically, the value would change, i.e, for the given example the median would be " 3 ", " 10 ", and " 10 " but these would all represent the third number in the original set, i.e., 3.
} 
analytical tools are; and what distortions of statistical outputs is associated with particular violations. There is much more work on the violation of distributional assumptions (homogeneity of variance etc) and correction for these errors than it is for the effects of the assumption of intervality. But again, as seen in recent work, the effects of this violation can be severe.

Where to go from here? From the choice of measurement and its philosophical basis, to procedural elements in this measurement like numerical mapping, to the data analysis itself (which statistical models to use) there are numerous issues influenced by the ordinal nature of the data gathered. So how do we solve the problem of ordinality?

Now the important link between measurement and analysis: the data may not know where they come from, but they give indications of their place of birth. Different patterns and variations in data give indirect evidence for plausible data generating processes (or parameters). Different scales of measurement would logically give us different data conditions and data patterns: the number of response categories would cap the possible values, affecting also the degree of normality (or possible normality; ex. 3 categories vs. 11 categories). Because the response options are not guaranteed to be equally spaced, this binning issue also causes single Likert items and short scales to be prone to heavy skewness, bimodality and other "non-ideal" data conditions

Data simulation sidesteps the measurement problem, at least adequately enough for our needs. Why? Because while different measurement positions have different substantive assumptions about reality (i.e whether or not Attribute $A$ actually exists and this affects indicators $L$ or whether variation in indicators $L$ are due to other factors) the ontology of $A$ is only as important as its effect on the data. Thus if we can recreate data from different 'measurement realities', then by focusing only on the data analysis we can parse out how the different issues of ordinality (especially as encountered by a linear model) along the line ultimately affects the statistical inference.

It's important to note there is a host of literature that deal with categorical data, and ordered categorical data in particular. These include the use ordinal odds ratios, multinomial response models, logistic regression among others (Agresti, 2002; Agrest, 2010) as well as latent variable methods (Pareto \& Casacci, 2015; Johnson \& Albert, 1999) and allowing for a multilevel structure in the data (Hedecker, 2015). But it's also important to contrast this with the sheer ubiquity, and almost universal of the use of standard linear models on Likert data (Liddell \& Kruschke, 2018)

If it is true that linear models can be used pragmatically on Likert data, then its performance must be comparably similar to methods that do take into account the ordinal nature of the data; given the same datasets. Thus, how standard linear models fare against ordinal models when used on 'ambiguously ordinal' Likert items and scales are important to examine.

Thus the proposed project aims primarily 1.) to see which data conditions of Likert items and scales the different statistical models work best on. A discussion of the data conditions and the analysis tools will be discussed below. Secondly, 2.) whether there are systematic discrepancies or patterns that result from fitting a metric model to ordinal Likert data (and whether there is a way to correct for this)

\section{EXAMPLE PROBLEMS IN REAL CASES}


Liddell and Kruschke (2018) provide an example of when an ordinal analysis (ordered-probit regression) and a standard linear model (t-test) provide contradictory statistical conclusions. Movie ratings from Amazon.com, which uses a 5-star system is the dependent variable. A 1 star review denotes that the viewer finds the movie very bad while a 5 star denotes that it is very good. Like Likert type scales, while intuitive, there is no guarantee that the intervals between response options are equal in interval (despite again, a standard linear model assuming it is so). 36 movies are analyzed in this manner, and some cases show a difference of conclusions between ordered probit and linear model.

However, this stark difference of outputs by the models need not be the case for all studies. Many studies use the ordered probit regression as a sensitivity analysis if they use the standard ordinary least squares regression, especially if the DV is a Likert scale. One such study by Nyhan, Porter, Reifler, \& Wood (2017), in a substantive experiment, tries to show if making individuals read 'correction' articles after reading 'fake news' changed the individuals belief in the original article, and whether they changed their belief regarding the candidates. While no data distribution summaries were presented, the authors report that all ordered probit regression results are similar to the OLS regression results.

In fact, prowling through accounts of methodologists who use ordered probit in their analyses, it seems that errors to the severity of Liddell and Kruchke's example (2018) might be rare. This is encapsulated in by Scholten (2011) who says that "inadmissible parametric statistics tend to agree with their more cumbersome admissible counterparts, so that real harm is rarely done by executing statistical analyses that are strictly inappropriate." However, as the Liddell \& Kruchke (2018) cheeckily state: "Arguing that it is okay to use a metric model instead of an ordered-probit model because a difference in conclusions may be rare is like arguing it's okay to drive drunk because accidents rarely happen"

The routine use of ordinal data analysis methods alongside traditional methods also outlines the importance of sensitivity analysis (Silberzahn, et al., 2018; van Dongen et al., 2018 see also 'multiverse' analysis: Steegen, Tuerlincky, Gelman, Vanpaemel, 2016). That is, whether different models, using more or less the same inputs, converge in their outputs. And if they do not, what the discrepancy reveal about the data, underlying relationships of the variables, study design and so on. Sensitivity and multiverse analysis also allows to probe the fragility of a study's conclusion to certain assumptions and analytic choices.

\section{LEARNING FROM ERROR}

The current proposal can also be seen as compatible with the error statistical philosophy (Mayo, 1996; Mayo, 2018). That is, how we bridge from what we can observe (sample data) to what we can't (theories, hypotheses, psychological constructs, parameters etc) is argued to rely on whether we can detect, correct and learn from our errors. Moving use from merely probable claims, to strong inferential well-probed ones. This error statistical approach demand that we ask "if we don't know when we are wrong, then how can we know when we are right?" and this extends not only to inferential claims but also inferential tools. (Mayo, 1996; Mayo, 2018)

And this perspective underlies the contribution of the project: when we know when our standard linear metric methods fails, we also know when it will work reliably. It can be thought of as similar 
to other issues of regression models; range restriction for instance. We know how to diagnose it, and therefore correct when it is present or design a study as to prevent it entirely.

As a meta-methodological project, the proposal aims to contribute 1.) to the production of knowledge. Knowing which models work in specific data conditions allow us to plan for good statistical inference (should we prefer ordered-probit in all instances? Is linear regression pragmatic and good enough, and when? Is permutation testing a good alternative? Does it even matter?) but also 2.) in the consumption of knowledge. If we see published literature use mismatched analytic tools for certain data conditions, how do we -whether informally or formally- reweight their conclusion? And what do the results of the sensitivity analysis tell us about the hypotheses/relationships we aim to inquire? (i.e How do make 'make our errors talk?' Mayo; 1996)

\section{DATA SIMULATION}

To achieve the study's objective, the main working horse would be data simulation. By doing a simulation, we can set our parameters (data generating processes) and thus set the properties of the different data distributions. In each of these produced datasets, we can then apply our different analytic tools/models (Traditional Linear, Ordered Probit, Logistic Regression, Randomization Inference, Rank Sum Tests) and see whether they recover the parameters that we set (the 'real effect size') and the correct statistical decision (significant if there are true effect sizes and non-significant if the null is true). By doing so, we see which tools work best in which data conditions. In other words, we explicitly create a universe where we know when and how our models work and fail.

The creation of these data with different distribution properties is paramount. however Issue: Differing the underlying data conditions (i.e data generating processes) for all elements: 1.) $N$, 2.) skew, 3.) kurtosis, 4.) error normality, 5.) homoscedasticity, 6.) number of categories, 7.) number of items, 8.) interval widths (binning) would lead to the production of around 25,920 to 187,500 simulated different datasets

Solution: instead of analyzing all conditions at once, this will be 'chunked' into relevant clusters. These clusters include:

1.) $N$, Number of scale categories, number of items, and normality [378 datasets]

a. $\mathrm{N}=20,50,100,200,500,100$

b. Number of categories: 5,7 and 9

c. Number of items: 4, 8, 12,16, 20, 50, 100

d. Normality: strong positive skew, normal, strong negative skew

2.) Skew, kurtosis, and homogeneity of variance [75 datasets]

a. Kurtosis: Strong leptokurtosis, mild leptokurtosis, normal, mild platykurtosis, strong platykurtosis

b. Skew: Strong positive skew, mild positive skew, normal, mild negative skew, strong negative skew

c. HOV: homogenous, *1.5 SD, *2 SD, *2.5 SD, *3 SD

3.) Binning, number of items, number of categories [60 datasets]

a. Binning: Unequal widths type 1 (high extreme widths, low central widths), type 2 (high central widths, low extreme widths), type 3 (high positive widths, low negative widths), type 4 (high negative widths, low positive widts), and equal widths

b. Number of items: $8,16.24$ 
c. Number of categories: 4, 5, 7, 9

4.) If in the first iteration, different clusters show different insights a $2^{\text {nd }}$ iteration of clusters can be made with these relevant variables

Sample Analysis Sheet: whether the analysis tools recover the true effect size $\left(d\right.$, epsilon $\left.{ }^{\wedge} 2, b\right)$ and keeps the false positive error rate below the nominal alpha level. The datasets and whether the analysis tools capture their properties will be summarized into a table for more efficient lookovers

\begin{tabular}{|c|c|c|c|c|c|c|c|c|}
\hline \multicolumn{9}{|c|}{ Sample Summary Analysis Table } \\
\hline \multicolumn{2}{|c|}{ Analysis Tools } & Condition 1 & Condition 2 & Condition 3 & Condition4 & Condition5 & Condition 6 & Condition 7 \\
\hline \multirow[b]{2}{*}{ Traditional } & Effect Size & $\checkmark$ & $\checkmark$ & $\checkmark$ & $\checkmark$ & $\mathrm{X}$ & $\mathrm{X}$ & $\mathrm{X}$ \\
\hline & $\begin{array}{c}\text { False positive } \\
\text { error rate }\end{array}$ & $\checkmark$ & $\checkmark$ & $\checkmark$ & $\mathrm{X}$ & $\mathrm{X}$ & $\mathrm{X}$ & $\mathrm{X}$ \\
\hline \multirow[b]{2}{*}{ Ordered Probit } & Effect Size & $\checkmark$ & $\checkmark$ & $\checkmark$ & $\checkmark$ & $\checkmark$ & $\checkmark$ & $\checkmark$ \\
\hline & $\begin{array}{c}\text { False positive } \\
\text { error rate }\end{array}$ & $\checkmark$ & $\sqrt{ }$ & $\checkmark$ & $\checkmark$ & $\checkmark$ & $\checkmark$ & $\mathrm{X}$ \\
\hline \multirow{2}{*}{$\begin{array}{c}\text { Logistic } \\
\text { Regression }\end{array}$} & Effect Size & $\ldots$ & $\ldots$ & $\ldots$ & $\ldots$ & $\ldots$ & $\ldots$ & $\ldots$ \\
\hline & $\begin{array}{c}\text { False positive } \\
\text { error rate }\end{array}$ & $\ldots$ & $\ldots$ & $\ldots$ & $\ldots$ & $\ldots$ & $\ldots$ & $\ldots$ \\
\hline \multirow[b]{2}{*}{$\begin{array}{l}\text { Randomization } \\
\text { Inference }\end{array}$} & Effect Size & $\ldots$ & $\ldots$ & $\ldots$ & $\ldots$ & $\ldots$ & $\ldots$ & $\ldots$ \\
\hline & $\begin{array}{c}\text { False positive } \\
\text { error rate }\end{array}$ & $\ldots$ & $\ldots$ & $\ldots$ & $\ldots$ & $\ldots$ & $\ldots$ & $\ldots$ \\
\hline \multirow{2}{*}{$\begin{array}{c}\text { Rank Sum } \\
\text { Tests }\end{array}$} & Effect Size & $\ldots$ & $\ldots$ & $\ldots$ & $\ldots$ & $\ldots$ & $\ldots$ & $\ldots$ \\
\hline & $\begin{array}{c}\text { False positive } \\
\text { error rate }\end{array}$ & $\ldots$ & $\ldots$ & $\ldots$ & 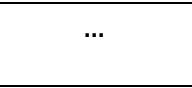 & $\ldots$ & $\ldots$ & $\ldots$ \\
\hline
\end{tabular}

The question becomes then: is the results of the data simulation generalizable? By making sure we include relevant parameters; the ones that we also actually see and concern ourselves in real data sets then the simulation should be appropriate and should hold-up. Further, to gain insights about our methods, there's very few other alternatives than simulation (mathematical proofs being another one), and while not the panacea, a well-done and exhaustive simulation should aid in our understanding of our tools. However, generalizability will always be a valid concern that is why, for Phase 2, a re-analysis of an actual dataset can also be done proof of concept of what is learned in the data simulation. 


\section{REFERENCE:}

Agresti, A. (2002). Categorical data analysis, New York: J.

Agresti, A. (2010). Analysis of ordinal categorical data (Vol. 656). John Wiley \& Sons.

Borsboom, D. (2018). The Philosophy of Psychometrics. Retrieved from https://vimeo.com/256145513

Casacci, S., \& Pareto, A. (2015). Methods for quantifying ordinal variables: a comparative study. Quality \& Quantity, 49(5), 1859-1872.

Field, A. (2017). Discovering Statistics Using IBM SPSS Statistics: North American Edition. SAGE.

Field, A. P., \& Wilcox, R. R. (2017). Robust statistical methods: A primer for clinical psychology and experimental psychopathology researchers. Behaviour research and therapy, 98, 19-38.

Hedeker, D. (2015). Methods for multilevel ordinal data in prevention research. Prevention Science, 16(7), 9971006.

Heeren, T., \& D'Agostino, R. (1987). Robustness of the two independent samples t-test when applied to ordinal scaled data. Statistics in medicine, 6(1), 79-90.

Hsu, T. C., \& Feldt, L. S. (1969). The effect of limitations on the number of criterion score values on the significance level of the F-test. American Educational Research Journal, 6(4), 515-527.

Johnson, V. E., \& Albert, J. H. (1999). Modeling ordinal data.

Kampen, J., \& Swyngedouw, M. (2000). The ordinal controversy revisited. Quality and quantity, 34(1), 87-102.

Keppel, G., \& Wickens, T. D. (2004). Design and Analysis: A Researcher's Handbook. Prentice Hall

Kline, R. B. (2015). Principles and practice of structural equation modeling. Guilford publications.

Knapp, T. R. (1990). Treating ordinal scales as interval scales: an attempt to resolve the controversy. Nursing research, 39(2), 121-123.

Liddell, T. M., \& Kruschke, J. K. (2018). Analyzing ordinal data with metric models: What could possibly go wrong?. Journal of Experimental Social Psychology, 79, 328-348.

Likert, R. (1932). A technique for the measurement of attitudes. Archives of Psychology, 22 140, 55.

Lord, F. M. (1953). On the Statistical Treatment of Football Numbers.

Marcus-Roberts, H. M., \& Roberts, F. S. (1987). Meaningless statistics. Journal of Educational Statistics, 12(4), 383-394.

Mayo, D. G. (1996). Error and the growth of experimental knowledge. University of Chicago Press.

Mayo, D. G. (2018). Statistical inference as severe testing: How to get beyond the statistics wars. Cambridge University Press.

Norman, G. (2010). Likert scales, levels of measurement and the "laws" of statistics. Advances in health sciences education, 15(5), 625-632.

Norman, G. (2010). Likert scales, levels of measurement and the "laws" of statistics. Advances in health sciences education, 15(5), 625-632.

Nunnally, J. C., \& Bernstein, I. H. (1967). Psychometric theory(Vol. 226). New York: McGraw-Hill.

Nunnally, J. C., \& Bernstein, I. H. (1994). Psychometric Theory (McGraw-Hill Series in Psychology) (Vol. 3). New York: McGraw-Hill.

Silberzahn, R., Uhlmann, E. L., Martin, D. P., Anselmi, P., Aust, F., Awtrey, E., ... \& Carlsson, R. (2018). Many analysts, one data set: Making transparent how variations in analytic choices affect results. Advances in Methods and Practices in Psychological Science, 1(3), 337-356.

Silberzahn, R., Uhlmann, E. L., Martin, D. P., Anselmi, P., Aust, F., Awtrey, E., ... \& Carlsson, R. (2018). Many analysts, one data set: Making transparent how variations in analytic choices affect results. Advances in Methods and Practices in Psychological Science, 1(3), 337-356.

Steegen, S., Tuerlinckx, F., Gelman, A., \& Vanpaemel, W. (2016). Increasing transparency through a multiverse analysis. Perspectives on Psychological Science, 11(5), 702-712.

Steegen, S., Tuerlinckx, F., Gelman, A., \& Vanpaemel, W. (2016). Increasing transparency through a multiverse analysis. Perspectives on Psychological Science, 11(5), 702-712.

Stevens, S. S. (1946). On the theory of scales of measurement. 
van Dongen, N. N. N., van Doorn, J., Gronau, Q. F., van Ravenzwaaij, D., Hoekstra, R., Haucke, M., ... Wagenmakers, E. (2018, December 6). Multiple Perspectives on Inference for Two Simple Statistical Scenarios. https://doi.org/10.31234/osf.io/ue5wb

van Dongen, N. N. N., van Doorn, J., Gronau, Q. F., van Ravenzwaaij, D., Hoekstra, R., Haucke, M., ... Wagenmakers, E. (2018, December 6). Multiple Perspectives on Inference for Two Simple Statistical Scenarios. https://doi.org/10.31234/osf.io/ue5wb

Wilcox, R. R. (2011). Introduction to robust estimation and hypothesis testing. Academic press.

Zand Scholten, A. (2011). Admissible statistics from a latent variable perspective. Unpublished doctoral dissertation. University of Amsterdam, Amsterdam.

Zand Scholten, A. (2011). Admissible statistics from a latent variable perspective. Unpublished doctoral dissertation. University of Amsterdam, Amsterdam. 